\title{
Thermalization in a Confining Gauge Theory at Strong Coupling
}

\author{
Takaaki Ishii, ${ }^{a}$ Elias Kiritsis ${ }^{* a, b}$ and Christopher Rosen ${ }^{a}$ \\ ${ }^{a}$ Crete Center for Theoretical Physics, University of Crete \\ Heraklion 71003, Greece \\ ${ }^{b}$ University of Paris Diderot, Sorbonne Paris Cite APC \\ Paris F-75205, France \\ E-mail: ishii@physics.uoc.gr, hep.physics.uoc.gr/ kiritsis, \\ rosen@physics.uoc.gr
}

\begin{abstract}
The equilibration dynamics of strongly interacting matter has a computationally favorable holographic description in terms of dynamical processes in higher dimensional gravity theories. One can exploit this holographic description to study the response of a strongly coupled confining gauge theory to time dependent perturbations by a relevant scalar operator. Gravitationally, this involves solving a set of non-linear Einstein-Dilaton equations subject to particular time dependent boundary conditions. The solutions to this system allow one to comment in some detail on the thermalization time for various perturbations, as well as to quantify the properties of the final state in terms of the perturbation parameters. We discuss the role of the dual gauge theory's confinement scale on these results, and highlight a realization of a previously anticipated universal scaling regime in the "abrupt quench" limit.
\end{abstract}

The European Physical Society Conference on High Energy Physics 22-29 July 2015

Vienna, Austria

${ }^{*}$ Speaker. 


\section{Overview}

Dynamical properties of strongly interacting matter are interesting both because of the theoretical challenges encountered in any attempt to quantify them, as well as the fact that they are increasingly accessible in the laboratory. One of the most reliable methods of performing computations in strongly coupled field theories is to regularize the theory on a lattice. This method, however, employs a Euclidean formulation of the field theory and hence it is difficult (if not impossible) to make contact with real-time dynamics. Finding a way to circumvent such obstacles takes special importance in the context of ongoing programs in relativistic heavy ion collisions. At the energy scales currently realized, the hot, dense matter produced in such collisions of heavy nuclei behaves as a strongly interacting plasma and thus novel theoretical approaches are needed to describe its behavior. One such approach is based on a holographic duality between gauge theories and string theories. This approach was employed in [1] to model the dynamical response of a confining gauge theory at strong coupling, a work which forms the basis for these proceedings.

\section{Thermalization in Holography}

Given any strongly coupled medium, a useful characterization of its properties is provided by its response to a variety of external perturbations. A quantum field theory effectively described by the Lagrangian $\mathscr{L}_{\mathrm{QFT}}$ couples to an operator $\mathscr{O}$ in a manner described by the source $f_{0}$ as

$$
\mathscr{L}_{\mathrm{QFT}} \rightarrow \mathscr{L}_{\mathrm{QFT}}+f_{0} \mathscr{O}
$$

Then if the source is isotropic in space and changes only in time, the probe will do work on the system as dictated by the following Ward identity:

$$
\nabla^{t}\left\langle T_{t t}\right\rangle=\dot{f}_{0}\langle\mathscr{O}\rangle
$$

Here the "dot" denotes a partial derivative with respect to time and $T$ is the stress-energy tensor.

The response of the system to the insertion of the time dependent perturbation can be measured in any of a number of distinct ways. A particularly natural option is provided by the one point functions of the various gauge invariant operators of the field theory. By examining the dependence of these expectation values on time, one can access both the endpoint of the dynamical evolution, as well as the route the system takes to arrive there.

It may happen that the late time endpoint of the evolution is independent of time, and the various one point functions take the form

$$
\langle\mathscr{O}(t \rightarrow \infty)\rangle \sim \operatorname{tr} \rho \mathscr{O},
$$

where $\rho$ is a thermal distribution. If this is the case, then the endpoint of the evolution is a thermal state, and the equilibration process is called "thermalization".

For strongly coupled field theories with a holographic dual the thermalization process can alternatively be described in terms of the weakly coupled bulk variables of a gravitational theory in one higher dimension. In terms of these variables, thermalization in a $3+1$ dimensional field theory is understood to be the same as black brane formation in a $4+1$ dimensional theory with gravity. 


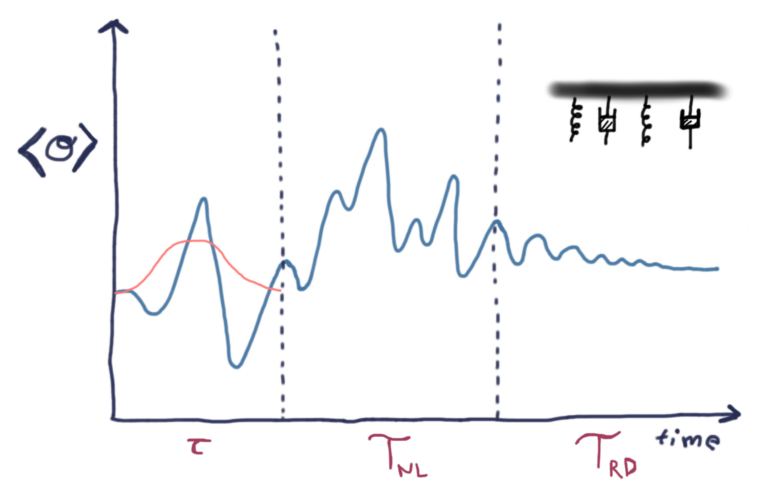

Figure 1: Generic response of a holographic system to a time dependent perturbation. After the time $\tau$ the system is driven out of equilibrium, and may pass through any number of non-linear regimes $\mathscr{T}_{\mathrm{NL}}$ before "ringing down" to thermal equilibrium with characteristic time scale $\mathscr{T}_{\mathrm{RD}}$. The ring-down regime is anticipated from the well known fact that black branes respond to small perturbations much like a system of damped oscillators (inset).

The formulation of the dynamical process in terms of the dual gravity theory offers benefits both in terms of computation and intuition. In figure 1, a generic expectation for the dynamical evolution of a holographic one point function is shown. To characterize the evolution, it is convenient to divide the response into different regimes controlled by characteristic time scales. For example, if one imagines varying the source $f_{0}$ over a timescale $\tau$, then $\tau$ roughly characterizes the amount of time taken to drive the system out of equilibrium. The subsequent non-equilibrium dynamics will evolve according to the non-linear gravitational equations of motion, and thus it could be possible that the system will pass through a highly non-linear regime, $\mathscr{T}_{\mathrm{NL}}$. This time scale may in principle be further subdivided into any number of distinct regimes.

Assuming a horizon forms in the bulk, and thus that the dual theory thermalizes at late times, it is natural to expect that a "ring-down" regime defines the system's near-equilibrium behavior. This expectation is based on the fact that the linearized response of the final state black brane to small perturbations is governed by the lowest lying quasi-normal mode in the spectrum at late times.

In light of the preceding discussion, one can define a thermalization time

$$
\mathscr{T}_{\text {THERM }}=\mathscr{T}_{\mathrm{NL}}+\ldots+\mathscr{T}_{\mathrm{RD}}
$$

as the sum of all the characteristic time scales defining the dynamic evolution except the time scale characterizing the duration of the applied external source. Thus defined, the thermalization time depends only on the non-equilibrium dynamics of the strongly coupled matter. In the majority of holographic examples of thermalization in a strongly coupled field theory, the thermalization time is dominated by the ring-down regime, i.e $\mathscr{T}_{\mathrm{THERM}} \approx \mathscr{T}_{\mathrm{RD}}$.

\section{Dynamical Quenches in a Confining Gauge Theory}

One ingredient missing from previous holographic investigations of thermalization is the presence of a confinement scale analogous to the $\Lambda_{\mathrm{QCD}}$ of quantum chromodynamics. It is natural to 
wonder if the existence of such an additional characteristic energy scale could lead to more complicated thermalization processes in the dual field theory. Dynamical response to perturbations in a confining gauge theory has been previously studied in the so-called hardwall model $[2,3]$. The hardwall model is a conceptually useful, but somewhat ad hoc implementation of holographic confinement in which the geometry is cut-off by hand at some slice in the radial direction. An obvious next step is to determine whether or not something similar can happen in more realistic holographic models of confining gauge theories. An example of one such class of models is the Einstein-Dilaton class, whose solutions arise from an action of the form

$$
S=\frac{1}{2 \kappa^{2}} \int \mathrm{d}^{5} x \sqrt{-g}\left(R-\frac{4}{3}(\partial \varphi)^{2}+V(\varphi)\right)-\frac{1}{\kappa^{2}} \int_{\partial} \mathrm{d}^{4} x \sqrt{-\gamma} \mathscr{K} .
$$

The second term in this action is the familiar Gibbons-Hawking-York boundary term, while the first term describes the interactions of a scalar field $\varphi$ coupled to gravity, subject to the scalar potential $V$.

In a "bottom up" holographic model, the scalar potential $V$ is arbitrary and can be tuned to induce the desired dual physics. In the present case, we will choose $V$ such that the dual boundary gauge theory is confining at low temperatures and such that solutions with non-zero scalar correspond to relevant deformations by a dimension three scalar operator (for details, see [1]). An example of a scalar potential that satisfies these conditions is given by

$$
V(\varphi)=\frac{12\left(1+a \varphi^{2}\right)^{1 / 4} \cosh \frac{4}{3} \varphi-b \varphi^{2}}{L^{2}}
$$

with the parameter choice $(a, b)=(1 / 500,10009 / 1500)$ and $L$ the AdS scale.

The solutions to this model describe the various phases available to the dual boundary theory. At zero temperature, the dual gravitational solution is a horizon-less geometry with a running scalar and a rather innocuous naked singularity deep within the throat. Ideally, we would like to perturb this geometry by varying the source for the dual scalar operator in time. In practice, the rapidly diverging bulk scalar complicates the numerical analysis and it is necessary to introduce an infrared cutoff deep in the throat region of the geometry.

One sensible option for introducing such a cutoff is to turn to another branch of solutions for the initial state. A particularly well suited branch contains the "small black hole" solutions, which shield the singularity behind a very small horizon. This horizon regulates the IR, but not without introducing some drawbacks. First, the introduction of a horizon in the initial state obviously renders any information related to horizon formation inaccessible. Second, the small black holes are never the thermodynamically preferred solutions in the holographic dual. Nonetheless, they have the distinct advantage of retaining much of the flavor of the zero temperature solution (for example a scalar which grows very large in the IR) while shielding the numerical methods from the existence of the IR singularity.

Roughly, our computational strategy will be as follows. Beginning from a small black hole initial state, we turn on a time dependent source of the form

$$
f_{0}(t)=\tilde{f}_{0}\left(1+\tilde{\delta} e^{-\frac{\nu^{2}}{2 \tilde{\tau}^{2}}}\right)
$$


which can be tuned by varying $\tilde{\delta}$ and $\tilde{\tau}$. This change in the scalar at the boundary propagates into the bulk, backreacting on the geometry. This process is encoded in the non-linear Einstein equations, which must be solved numerically time step by time step until a final steady state solution has been obtained. At each time step, a standard application of the holographic dictionary allows one to read off the one-point functions in the dual gauge theory.

The response reveals an energy density which changes markedly on the time scale of the quench $\tilde{\tau}$, followed by a rapid equilibration to its final state value. To gain a better understanding of the rapid approach to thermal equilibrium, it is useful to look into the late time response of the system. In figure 2 the evolution of the scalar one point function is plotted on a logarithmic scale. One notes immediately that after a short time (again controlled by the quench width $\tilde{\tau}$ ), the system oscillates with a well defined frequency $\omega_{*}$ while simultaneously approaching its equilibrium value exponentially with decay constant $\Gamma$. This is exactly as one might expect from a linear system controlled by an excited mode of the form $\omega_{1}=\omega_{*}-i \Gamma$. Evidently, even in the confining model of (3.1-3.2) the thermalization time appears to be dominated by the linear regime, $\mathscr{T}_{\text {THERM }} \approx \mathscr{T}_{\text {RD }}$.

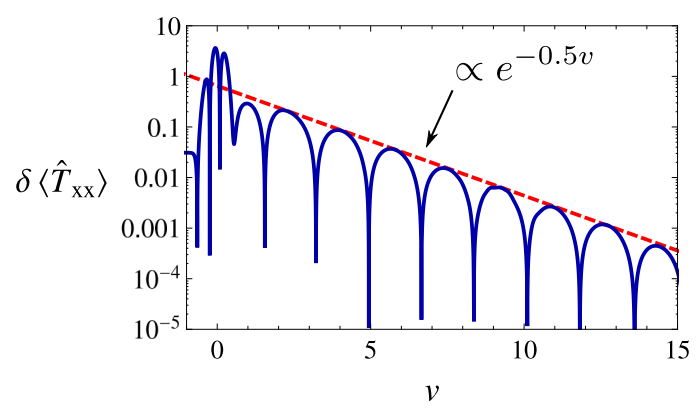

Figure 2: Typical late-time behavior of the magnitude of a one-point function's deviation from its equilibrium value. After the quench time $\sim \tilde{\tau}$ the response equilibrates like a damped oscillator. The damped oscillations correspond to the excitations of the gravitational system's lowest quasi-normal mode.

Beyond thermalization times, the holographic approach also provides some insight into the dependence of the final state on parameters of the quench. In figure 3 the final state energy density is plotted as a function of quench duration with fixed (large) amplitude, and as a function of quench amplitude at fixed (short) duration. The appearance of simple power law scaling regimes is pronounced. Combining the results of the two plots, one finds that in the limit of abrupt quenches (those whose quench width is much smaller than all other dimensionful scales)

$$
\left\langle T_{t t}\right\rangle_{\mathrm{FINAL}} \sim\left(\frac{\tilde{\delta}}{\tilde{\tau}}\right)^{2} .
$$

In fact this simple scaling relation was already anticipated on general grounds, and is a special case of the universal scaling formula in [4] for a dimension three scalar operator. That the scaling should be universal readily follows from the fact that for very fast quenches, the perturbation does not have time to propagate far from the boundary before the quench terminates. Since the near boundary region of many holographically relevant spacetimes is asymptotically AdS, abrupt quenches are indifferent to the IR features of the geometry distinguishing different holographic spacetimes. 

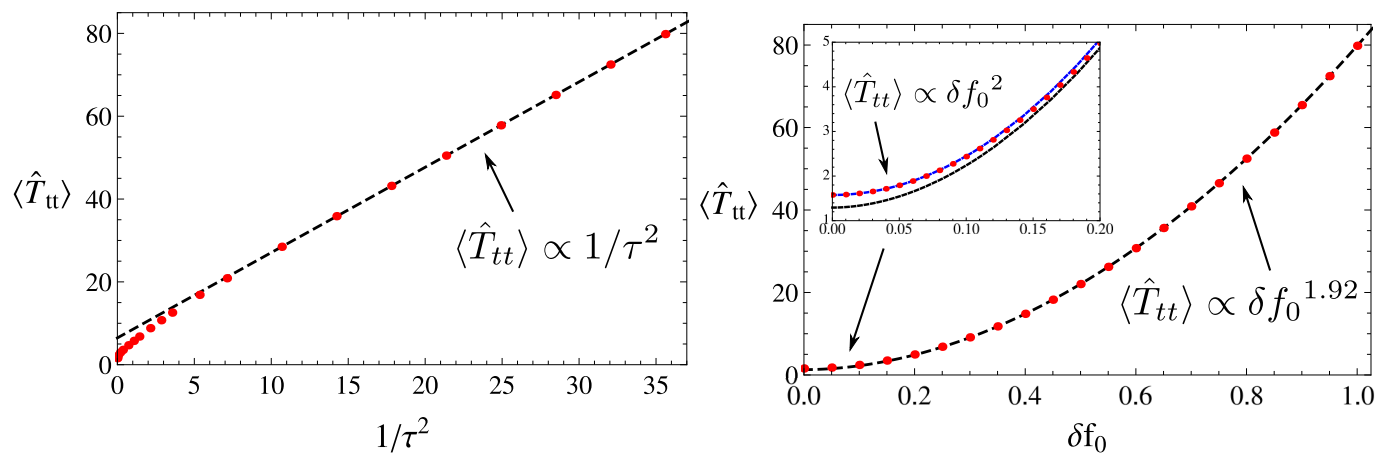

Figure 3: Final state energy density $\left\langle T_{t t}\right\rangle$ as a function of quench parameters. In the left plot the quench amplitude is held fixed, while in the right plot the quench duration is fixed.

\section{Discussion}

Perhaps the most important observation from our analysis is that in every quench we performed, the thermalization time continued to be dominated by the linear response time scale. Thus, even the presence of a confinement scale was insufficient to induce a new non-linear time scale in the dynamical response. This might be a consequence of our choice of initial state. For technical reasons alluded to before, our numerical stability suffers as we decrease the size of the initial state black hole. One might expect that the confinement scale becomes important to the dynamical response when it is large compared to all other energy scales in the problem. The smallest black hole we can reliably perturb has an energy density comparable to the confinement scale. In other words $\left\langle T_{t t}\right\rangle / f_{0}{ }^{4} \sim O(1)$. Accordingly, it is perhaps not so surprising that we see no deviation from the ubiquitous quasi-normal mode driven thermalization familiar from other holographic models.

Ultimately, we would like to perform these dynamical quenches in initial states with $\left\langle T_{t t}\right\rangle / f_{0}{ }^{4} \ll$ 1 , and ideally in the zero temperature solution itself. It is here that we expect the response to the probe to be most sensitive to the diverging scalar potential and the effects of the confinement scale in the dual theory. Understanding whether or not the properties of the scalar potential are sufficiently similar to the hardwall to give rise to scattering solutions which never thermalize would be very interesting. If such scattering solutions exist, identifying the location in parameter space which separates perturbations which thermalize from those that do not would permit a scaling analysis analogous to that performed in the well known case of the Choptuik phenomena [5].

Finally, it may be interesting to explore other probes of the thermalization process in this holographic theory. One obvious class of candidates are non-local probes such as Wilson loops and two-point correlators of large dimension operators. These probes share the feature that their holographic computation involves the study of bulk worldlines or worldsheets that sag into the bulk spacetime. The depths they probe in the geometry are controlled by the boundary separations of operators in space and time. In this way these probes are capable of sampling the response of the gravitational system at various locations in the radial direction, thus characterizing the equilibration process of the field theory at different length scales. 


\section{References}

[1] T. Ishii, E. Kiritsis, and C. Rosen, Thermalization in a Holographic Confining Gauge Theory, JHEP 08 (2015) 008 [arXiv: 1503.07766 ].

[2] B. Craps, E. Kiritsis, C. Rosen, A. Taliotis, J. Vanhoof, et al., Gravitational collapse and thermalization in the hard wall model, JHEP 1402 (2014) 120, [arXiv: 1311 . 7560].

[3] B. Craps, E. Lindgren, A. Taliotis, J. Vanhoof, and H.-b. Zhang, Holographic gravitational infall in the hard wall model, Phys.Rev. D90 (2014), no. 8 086004, [arXiv: 1406.1454 ].

[4] A. Buchel, R. C. Myers, and A. van Niekerk, Universality of Abrupt Holographic Quenches, Phys.Rev.Lett. 111 (2013) 201602, [arXiv: 1307 . 4740].

[5] M. W. Choptuik, Universality and scaling in gravitational collapse of a massless scalar field, Phys.Rev.Lett. 70 (1993) 9-12. 